\title{
Possible modulatory effects of male cues and social system on luteinizing hormone and pituitary sensitivity in two African mole-rats
}

\author{
N.C. Bennett* \& H. Lutermann \\ Mammal Research Institute, Department of Zoology and Entomology, University of Pretoria, \\ Private Bag X20, Hatfield, Pretoria, 0028 South Africa \\ Received 26 January 2012. Accepted 18 April 2012
}

\begin{abstract}
The current study set out to investigate the potential effects of olfactory stimuli on the basal circulating levels of luteinizing hormone (LH) and the response of the pituitary to an exogenous gonadotropin-releasing hormone (GnRH) challenge in two mole-rat species with induced ovulation but contrasting mating and social systems. Females were either kept in isolation, allowed only olfactory and auditory contact or in physical contact with a male. Basal LH as well as pituitary challenged LH blood samples were collected after six hours. Post-challenge LH levels were significantly elevated compared to baseline levels in both species. However, neither basal nor post-challenge LH levels differed significantly between treatments for females of either species. At the same time, while baseline LH was comparable between the two species, post-challenge levels were significantly greater for the solitary species. Our findings negate a modulatory role of male cues on LH levels but suggest increases in pituitary sensitivity in the solitary species. This could enhance reproductive success in species with only sporadic encounters between the sexes and merits further studies in other species.
\end{abstract}

Key words: Bathyergidae, induced ovulation, luteinizing hormone.

There are two patterns of ovulation that arise in mammals generally, and in mole-rats specifically, these being spontaneous and induced (Milligan 1975; Soulsbury \& Iossa 2010; Faulkes et al. 2010). Spontaneous ovulation arises from a continuous cycle of follicular development, whereby primordial follicles mature into Graafian follicles, which rupture to release the ovum (oocyte) following a spike in luteinizing hormone (LH). Post-ovulation, the Graafian follicle develops into a corpus luteum which is under the control of cyclical production of hormones (Milligan 1980). Induced ovulators experience a similar process of follicular growth and development to that of spontaneous ovulators but sensory stimulation of the vagina and cervix during copulation by a male is required for the production of ovarian hormones and ovulation to occur (Milligan 1980). Additionally, induced ovulators do not show steroid-induced pre-ovulatory LH surges. It has been suggested that the positive feedback mechanisms of steroid hormones on gonadotrophin release are either not present at all, or considerably reduced in induced ovulating females (Bakker \& Baum 2000).

Conaway (1971) proposed that the pattern of

*Author for correspondence. E-mail: ncbennett@zoology.up.ac.za ovulation should be viewed rather as a continuum, whereby induced and spontaneous ovulation are considered as two extremes at either end of this spectrum, with many species falling somewhere between the two.

The role of olfactory cues to the mating-induced pre-ovulatory surge in LH is not clear. In the ferret, an induced ovulator, there is some evidence to suggest that olfactory cues may be necessary for mating-induced pre-ovulatory surge in the $\mathrm{GnRH}$ neurons of the medial basal hypothalamus (MBH) (Bibeau et al. 1991). By contrast, Wersinger \& Baum (1997) found in the rabbit that while mating with intromission significantly increased the number of GnRH neurons in the MBH-expressing Fos-IR, male odours by themselves did not bring about this effect.

In the current study, we examined the basal circulating LH concentrations and the pituitary sensitivity to exogenous $\mathrm{GnRH}$ in female solitary Cape mole-rats and social Natal mole-rats in relation to the effect of chemical and physical stimulation with males. Both species are reported to show induced ovulation (Jackson \& Bennett, 2005; van Sandwyk \& Bennett 2005). In addition, significant increases in baseline LH but not the incremental 
response to a GnRH challenge during winter compared to summer have been observed for both species, suggesting that they retain their breeding potential throughout the year (Oosthuizen \& Bennett 2007; Oosthuizen et al. 2008). This study was undertaken in an attempt to discern if chemical cues alone could enhance LH and subsequent corpora lutea production. Our a priori prediction was that circulating LH and the pituitary response to an exogenous GnRH challenge would be higher in the females that are paired with a vasectomized male. We furthermore hypothesized that baseline $\mathrm{LH}$ and the response of the pituitary to a GnRH challenge would be elevated in Cape and Natal mole-rat females housed in chemical contact of a male when compared to control females.

Molteno (1999) found that the relationship between mean GnRH-stimulated LH concentration in reproductive and non-reproductive females was similar at three concentrations of exogenous $\mathrm{GnRH}(0.5,1.0$ and $2.0 \mu \mathrm{g})$. We thus chose to use the largest dose of GnRH. Since this was a oneoff experiment and could not be repeated because of the difficulty in obtaining sufficient animals, we chose to use the maximal dose for the challenge to ensure maximal release of LH from the pituitary luteotrophs.

All mole-rats used in this study were captured using modified Hickman live-traps (Hickman 1979). Eight males of each study species were collected from the Darling region of the Western Cape Province $\left(33^{\circ} 22^{\prime} \mathrm{S} 15^{\circ} 25^{\prime} \mathrm{E}\right.$, Cape mole-rats) and at Glengarry Park in the Kamberg region of the KwaZulu-Natal Midlands $\left(25^{\circ} 58^{\prime} \mathrm{S} 21^{\circ} 49^{\prime} \mathrm{E}\right.$, Natal mole-rats), respectively. Half of the males from each species were vasectomized three months prior to being placed in direct physical contact with the females by removing the vas deferens and epididymis from each testis under iso-fluorane gas induction (Jackson \& Bennett 2005). Vasectomy was only performed in males to be placed into physical contact with a female to prevent fertilization taking place, such that any resulting corpora lutea would be of ovulation and not pregnancy. A period of three months ensured that no spermatozoa were present in the vas deferens.

Eleven female Cape mole-rats and 12 female non-reproductive Natal mole-rats were captured at the same localities as the males during early winter and housed separately in plastic crates $(60 \mathrm{~cm} \times 30 \mathrm{~cm} \times 30 \mathrm{~cm})$ under constant laboratory conditions at $25.0 \pm 1^{\circ} \mathrm{C}$ and on a controlled light cycle and fed ad libitum for a period of five weeks prior to the experiments. This period was chosen to coincide with the length of an average oestrus cycle in bathyergids of approximately one month (Faulkes et al. 1990, 2010).

For the purpose of the experiment females were divided into three groups of four females from each species for a period of five weeks. The first group were control females that were kept separately without the presence of a male. Owing to a shortage of females, this group contained only three females of Cape mole-rats. In the second group, females were housed in the presence of a nonvasectomized male but separated by a metal grid, allowing chemical and auditory, but not physical contact. In the last group females were housed with a vasectomized male, allowing physical contact and coitus to take place. Male access was permanent for Natal mole-rat females but limited to several hours per day for Cape mole-rats to prevent physical injuries in this highly xenophobic species.

Studies investigating the response of the pituitary to a GnRH challenge following pairing up in an induced ovulator are rare indeed. In rabbits the LH surge following copulation is maximal at 2-4 hours after coitus (Hillard et al. 1964). We selected a time period of six hours post-coitus to challenge the pituitary in the hope that we would see an elevation in LH following successful coitus and the LH surge, and assumed this time period would be suitable for the comparison of mole-rats in physical contact compared to those housed on their own or in the presence of a male but not allowed physical contact.

In all experiments $2 \mu \mathrm{g}$ of GnRH (synthesized in the laboratory of R.P. Millar) in $200 \mu$ l saline was administered subcutaneously. Blood samples of $300-400 \mu \mathrm{l}$ were taken from foot vein of the adult females after vasodilatation in a temperatureregulated chamber at $36^{\circ} \mathrm{C}$ for $20 \mathrm{~min}$ immediately before and $20 \mathrm{~min}$ after a single subcutaneous injection. All blood sampling was performed between 9:00 and 13:00.

The LH concentrations were measured and validated in an in vitro bioassay based on the production of testosterone by mouse Leydig cells as described in van Damme et al. (1974). Sensitivity of the assay (determined at $90 \%$ binding) was $12.7 \mathrm{mI} \mathrm{U} /$ tube or $2.5 \mathrm{mI} \mathrm{U} / \mathrm{ml}$. Intra- and interassay coefficients of variation for repeated measurement of a quality control were $8 \%(n=4)$ and $11 \%(n=8)$ respectively. 


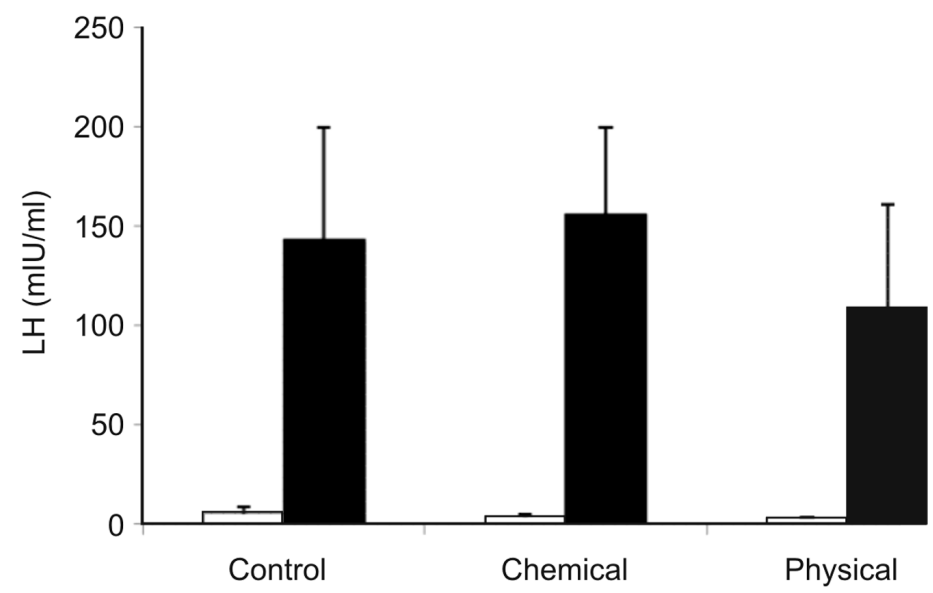

Fig. 1. Pre- and post-GnRH-challenge LH levels (mIU) of Cape mole-rats (Georychus capensis) for isolated (i.e. control) females and those in chemical and physical contact with conspecific males. Depicted are mean \pm S.E. Open bars indicate pre-challenge and filled bars post-challenge values.

Females were sacrificed with an overdose of halothane (AstraZeneca, Wilmington, Delaware). Ovaries were dissected out and placed in Bouin's fixative for 24 hours and then transferred to $70 \%$ ethanol. After several washes in alcohol and subsequent dehydration, samples were embedded in paraffin wax. The ovary was sectioined $4 \mu \mathrm{m}$ thick, mounted on slides and stained in haematoxylin and eosin dye prior to microscopic examination for corpora lutea (Drury \& Wallington 1967).

Hormone data did not satisfy the criteria of a parametric distribution and normalization could not be achieved by transformation. Hence, we analysed data by employing a generalized estimation equation assuming a gamma-distribution and a log-link function with LH-levels as dependent variable. We included mole-rat species, condition (i.e. control, chemical or physical contact with a male conspecific) and time (i.e. pre- or postchallenge) as independent factors. All two- and three-way interactions were included in the model. For the comparison of the number of corpora lutea between the two study species, $t$-tests were employed. The statistical analysis was carried out with SPSS (Version 19.0, Chicago, Ill.) and data are presented as means \pm S.E.

All animals were collected under permit from the relevant conservation authorities and the experiments were approved by the Animal Use and Care Committee of the University of Pretoria (EC20624-012).

The pre- and post-challenge LH-levels for Cape and Natal mole-rats are displayed in Figs 1 and 2, respectively. On average, LH levels were signifi- cantly greater for Cape mole-rats compared with Natal mole-rats (Wald- $\chi^{2}=46.13$, d.f. $=1, P<$ $0.0001)$. By contrast, there was no significant general effect of the different conditions (i.e. control, chemical or physical) on LH values (Table 1). However, pre-challenge values were significantly lower than post-challenge ones (Wald- $\chi^{2}=306.15$, d.f. $=1, P<0.0001)$. Neither the interaction between species and condition nor the interaction between condition and time were significant (Table 1). By contrast, there was a significant interaction between species and time (Wald- $\chi^{2}=$ 130.80 , d.f. $=1, P<0.0001$ ) with baseline LH levels not differing significantly between Cape $(3.9 \pm$ $0.9 \mathrm{mIU} / \mathrm{ml}$ ) and Natal mole-rat females (3.9 \pm $0.4 \mathrm{mIU} / \mathrm{ml}$, LSD: d.f. $=1, \mathrm{P}=0.949)$. However, LH levels were significantly greater for Cape $(134.3 \pm 26.3 \mathrm{mIU} / \mathrm{ml})$ compared to Natal mole-rats after the GnRH-challenge $(8.3 \pm$ $1.0 \mathrm{mIU} / \mathrm{ml}$, LSD: d.f. $=1, P<0.0001)$. For both species, the post-challenge LH levels were signifi-

Table 1. Results of the generalized linear model evaluating the effects of species, condition and time on $\mathrm{LH}$ levels.

\begin{tabular}{lrrc}
\hline Variable & Wald- $\chi^{2}$ & d.f. & $P$ \\
\hline Species & 46.130 & 1 & $<0.0001$ \\
Condition & 0.244 & 2 & 0.885 \\
Time & 307.153 & 1 & $<0.0001$ \\
Species $\times$ condition & 3.772 & 2 & 0.152 \\
Species $\times$ time & 130.801 & 1 & $<0.0001$ \\
Condition $\times$ time & 1.195 & 2 & 0.550 \\
Species $\times$ condition $\times$ time & 1.170 & 2 & 0.557 \\
\hline
\end{tabular}




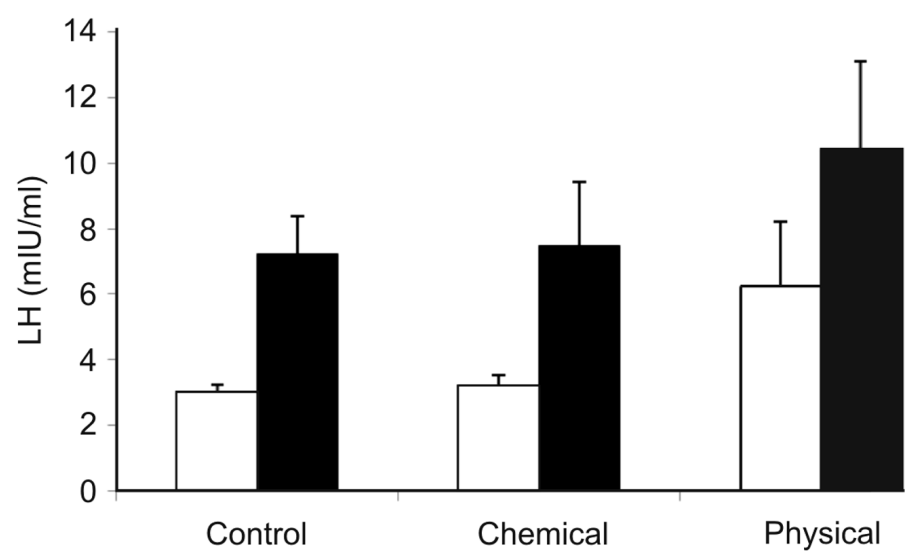

Fig. 2. Pre- and post-GnRH-challenge LH levels (mIU) of Natal mole-rats (Cryptomys hottentotus natalensis) for isolated (i.e. control) females and those in chemical and physical contact with conspecific males. Depicted are mean \pm S.E. Open bars indicate pre-challenge and filled bars post-challenge values.

cantly elevated compared to the baseline levels (LSD: d.f. $=1, P<0.0001$ for both species). The three-way interaction was not significant (Table 1). Only females in physical contact with males had corpora lutea in their ovaries (Table 2). With an average of $2.0 \pm 1.4$ and $2.25 \pm 1.3$ for Cape and Natal mole-rat females, respectively, the number of corpora lutea did not differ significantly between species $(t=-0.264$, d.f. $=6, P=0.801)$.

Contrary to our predictions we did not find any effect of either chemical or physical cues from a conspecific male on either baseline LH or LH responses to a GnRH challenge. Male cues have no priming effect. The observed occurrence of corpora lutea in both species where physical contact was allowed confirmed induced ovulation by copulation to bring about the LH surge. However, it has been shown in rabbits that the LH surge resulting from coitus is almost instantaneous and the LH levels are back to baseline within two to four hours

Table 2. Number of corporalutea found in female Cape and Natal mole-rats kept either under control conditions or in chemical or physical contact with a male conspecific, respectively.

\begin{tabular}{lcccc}
\hline Species & Female & Control & Chemical & Physical \\
\hline Cape mole-rat & 1 & 0 & 0 & 0 \\
& 2 & 0 & 0 & 2 \\
& 3 & 0 & 0 & 3 \\
Natal mole-rat & 4 & - & 0 & 3 \\
& 1 & 0 & 0 & 4 \\
& 3 & 0 & 0 & 2 \\
& 4 & 0 & 0 & 1 \\
& & 0 & 0 & 2 \\
\hline
\end{tabular}

(Hillard et al. 1964). This immediate release of LH suggests that there is a neuronal activation responsible for $\mathrm{LH}$ release and consequently the initial pituitary discharge of LH as well as FSH soon terminates after the mating event (Orstead et al. 1988). In the current study on mole-rats, blood samples were taken more than four hours after copulation which might have been too late to capture similar LH surges. Hence, male cues may affect short-term but not overall plasma LH levels, irrespective of the social or mating system of the study species.

Despite comparable baseline LH levels the increments of $\mathrm{LH}$ increases were more than 16 times larger for Cape compared to Natal mole-rat females after GnRH LH administration. This may indicate a greatly enhanced pituitary sensitivity in the former compared to the latter species. Although Cape mole-rat females exhibit increased basal levels of LH during the breeding season in which the study animals have been sampled the incremental response to GnRH challenges does not vary seasonally (Oosthuizen \& Bennett 2007). However, unlike Natal mole-rats, they do not maintain long-term contacts with males and are indeed highly xenophobic. Owing to the subterranean life-style of mole-rats, breeding dispersal requires energetically costly digging activity and is limited to wet periods (Bennett \& Jarvis 1988). An increased pituitary sensitivity may thus be adaptive for Cape mole-rats to ensure that a mating encounter with a male does indeed result in pregnancy. By contrast, Natal mole-rats live in permanent social groups and females will breed continuously throughout the year once they have 
found a mate. They are hence less constrained in their access to a suitable male for reproduction and may consequently experience less selective pressure to develop mechanisms that ensure successful ovulation. Our findings thus suggest that a species' mating system may exert significant effects on pituitary sensitivity to GnRH. There are currently no other studies comparing $\mathrm{LH}$ responses to GnRH challenges in species with different mating systems but our results indicate that this hypothesis deserves further attention in future studies.

\section{ACKNOWLEDGEMENTS}

The research was funded by the National Research Foundation. N.C.B. acknowledges funding for a DST-NRF SARChI Chair of Mammalian Behavioural Ecology and Physiology. H.L. acknowledges a postdoctoral research fellowship from the University of Pretoria.

\section{REFERENCES}

BENNETT, N.C. \& JARVIS, J.U.M. 1988. The reproductive biology of the Cape mole-rat, Georychus capensis (Rodentia, Bathyergidae). Journal of Zoology, London 214: 95-106.

BINEAU, C.E., TOBET, S.A., ANTHONY, E.L.P., CARROLL, R.S., BAUM, M.I. \& KING, J.C. 1991. Vagino-cervical stimulation of ferrets induces release of luteinizing hormone-releasing hormone. Journal of Neuroendocrinology 3: 29-36

DRURY, R.A.B. \& WALLINGTON, E.A. 1967. Carleton's Histological Technique. Oxford University Press, Oxford, U.K.

FAULKES, C.G., SICHILIMA, A.M., VAN SANDWYK, J, LUTERMANN, H. \& BENNETT, N.C. 2010. Control of ovulation in female giant mole-rats, Fukomys mechowii (Rodentia: Bathyergidae). Journal of Zoology, London 282: 64-74

FAULKES, C.G., ABBOTT, D.H. \& JARVIS, J.U.M. 1990. Social suppression of ovarian cyclicity in captive and wild colonies of naked mole-rats, Heterocephalusglaber. Journal of Reproduction and Fertility 88: 559-568.
HILLIARD, J., HAYWARD, J.N. \& SAWYER, C.H. 1984. Post-coital patterns of secretion of pituitary gonadotrophin and ovarian progestin in the rabbit. Endocrinology 75: 957-963.

JACKSON, C.R. \& BENNETT, N.C. 2005. Is the Natal mole-rat (Cryptomys hottentotus natalensis) a spontaneous or induced ovulator? Journal of Mammalogy 86: $1-6$.

MILLIGAN, S.R. 1975. Mating ovulation and corpus luteum function in the vole, Microbus agrestis. Journal of Endocrinology 42: 35-44.

MILLIGAN, S.R. 1980. Pheromones and rodent reproductive physiology. Symposium of the Zoological Society of London 45: 251-275.

MOLTENO, A.J. 1999. Reproductive regulation in female Damaraland mole-rats, Cryptomys damarensis: physiological and neuroendocrine mechanisms. Ph.D. thesis, University of Pretoria, Pretoria, South Africa.

OOSTHUIZEN, M.K. \& BENNETT, N.C. 2007. LH responses to single dose of exogenous $\mathrm{GnRH}$ in the Cape mole-rat (Georychus capensis): the pituitary potential for opportunistic breeding. Journal of Zoology, London 271: 198-202.

OOSTHUIZEN, M.K., BENNETT, N.C., LUTERMANN, H. \& COEN, C.W. 2008. Reproductive suppression in subordinate Natal mole-rats (Cryptomys hottentotus natalensis.): the pituitary response to exogenous $\mathrm{GnRH}$. General Comparative Endocrinology 159: 236-240.

ORSTEAD, K.H., HESS, D.L. \& SPIES, H.G. 1988. Pulsatile patterns of gonadotropins and ovarian steroids during estrus and pseudopregnancy in the rabbit. Biology of Reproduction 38: 733-743.

VAN DAMME, M.P., ROBERTSON, D.M. \& DICZFALUSY, E. 1974. An improved in vitro bioassay for measuring luteinizing hormone (LH) activity using mouse Leydig cell preparations. Acta Endocrinologia 77: 655-671.

VAN SANDWYK, J.d.T. \& BENNETT, N.C. 2005. Do solitary, seismic signalling Cape mole-rats, Georychus capensis, demonstrate spontaneous or induced ovulation? Journal of Zoology, London 267: 75-80.

WERSINGER, S.R. \& BAUM, M.I. 1997. Sexually dimorphic processing of somatosensory and chemosensory inputs to forebrain luteinizing hormone-releasing hormone neurons in mated ferrets. Endocrinology 138: $1121-1129$.

Responsible Editor: J.H. van Wyk 